\title{
Ambipolar Current Suppression, Analog/RF and Linearity Performance Improvement of Dual Material Stacked Gate Oxide TFET with Dielectric Pocket
}

dharmender nishad

Jaypee Institute of Information Technology

kaushal Nigam ( $\square$ kaushal.nigam@jiit.ac.in )

Jaypee Institute of Information Technology

\section{Research Article}

Keywords: Dual material, Ambipolarity, Dielectric pocket, Auxiliary gate, Control gate, Linearity

Posted Date: September 28th, 2021

DOl: https://doi.org/10.21203/rs.3.rs-741109/v1

License: (c) (i) This work is licensed under a Creative Commons Attribution 4.0 International License. Read Full License 


\title{
Ambipolar current Suppression, Analog/RF and Linearity Performance Improvement of Dual Material Stacked Gate Oxide TFET with Dielectric Pocket
}

\author{
Dharmender · Kaushal Nigam
}

Received: date / Accepted: date

\begin{abstract}
In this article, the impact of high-K and low-K dielectric pockets on $\mathrm{DC}$, analog/RF, and linearity performance parameters of dual material stacked gate oxide-dielectric pocket-tunnel field-effect transistor (DMSGO-DP-TFET) is investigated. In this regard, a stacked gate oxide $\left(\mathrm{SiO}_{2}+\mathrm{HfO}_{2}\right)$ with workfunction engineering is taken into consideration to improve the ON-state current $\left(I_{O N}\right)$, and suppress the ambipolar current $\left(I_{a m b}\right)$. To further improve the performance of the device, a high-K dielectric pocket $\left(\mathrm{HfO}_{2}\right)$ is used at the drain-channel interface to suppress the $I_{a m b}$, and at the source-channel interface a low-K dielectric pocket is used to improve the $I_{O N}$ and analog/RF performance. Moreover, length of stacked gate segments $\left(L_{1}, L_{2}, L_{3}\right)$, pocket height, and thickness are optimized to attain better $I_{O N} / I_{O F F}$ ratio, and suppress the $I_{a m b}$ which helps to achieve higher gain and design of analog/RF circuits. The DMSGO-DP-TFET outperforms the dual material control gate-dielectric pocketTFET (DMCG-DP-TFET) with SiO2 gate oxide and shows increment in $I_{O N} / I_{O F F}(\sim 4.23$ times $), 84 \%$ increment in transconductance $\left(g_{m}\right), 136 \%$ increment in cut-off frequency $\left(f_{T}\right), 126 \%$ increment in gainbandwidth-product (GBP), and better linearity performance parametrs such as $g_{m 2}, g_{m 3}$, VIP2, VIP3 and IIP3 making the proposed device useful for low power and radio frequency applications.
\end{abstract}

Keywords Dual material · Ambipolarity · Dielectric pocket $\cdot$ Auxiliary gate $\cdot$ Control gate $\cdot$ Linearity

Kaushal Nigam (Corresponding author) Department of Electronics and Communication Engineering, Jaypee Institute of Information Technology, Noida, U.P., India

E-mail: kaushal.nigam@jiit.ac.in

\section{Introduction}

MOSFETs are widely used for low-power switching, analog/RF design, and wireless communication systems. To further improve the performance and increase the packing density, MOSFET dimensions are aggressively down-scaling $[1,2]$. This inevitably results in some critical issues, such as high subthreshold leakage current, short channel effects (SCEs), and SS restriction of 60 $\mathrm{mV} /$ decade $[3,4]$. These challenges make conventional MOSFETs unfavorable for future low power switching and analog/RF applications.

To address the above issues, an alternate device structure based on quantum tunneling-FET (TFET) is considered one of the possible replacements of MOSFETs for low-power switching circuits due to lower leakage current, steeper SS below $60 \mathrm{mV} /$ decade and immunity to SCEs [5]-[7]. These features make TFETs more favourable for low-power, energy-efficient circuits. However, the major limitations of this device is inferior $I_{O N}$ and ambipolar behavior [8]. TFETs can be used in digital complementary logic circuits and high-frequency applications only if the ambipolar current of the device is suppressed. Ambipolar current implies the flow of current for both positive and negative $V_{G S}$ [9]. This happens as the tunneling junction is transferred from source to drain side by applying $V_{G S}<0$ (in case of ntype TFET). Because of ambipolar current, SS degradation and efficiency of the device for the complimentary digital logic circuits and high-frequency applications is limited $[10,11]$.

The ON-current of the TFETs is inferior due to inadequate inter band tunneling rate at the source junction. In TFET, high lateral electric field across the source junction is needed to lower the tunneling width and improve the tunneling current. As per the Wentzel- 
Kramers-Brillouin method, the BTBT probability depends exponentially on the width of the tunneling barrier, effective bandgap, the effective mass of charge carrier, and the energy band overlap [24].

In recent literature, researchers have made numerous efforts and proposed different methods to suppress the ambipolar current, improve the DC, analog/RF and linearity performance. Raad et al. [12] proposed a TFET based on hetero dielectric and workfunction engineering (HGD-DW TFET) and reported an $I_{O N} / I_{O F F}$ ratio of $\left(9.8 \times 10^{11}\right), g_{m}$ of $0.290 \mathrm{mS}, f_{T}$ as $\left(\sim 59.6 \mathrm{GH}_{Z}\right)$. Kondekar et al. [13] proposed TFET based on electrically doping (ED-TFET) to enhance the analog and radio frequency performance and reported an $I_{O N} / I_{O F F}$ ratio of $\left(\sim 10^{12}\right), g_{m}$ as $1.02 \mathrm{mS}, f_{T}$ of $\left(190 G H_{Z}\right)$. Nigam et al. [14] proposed a charge plasma based TFET (DMCG-CPTFET) and obtained an $I_{O N} / I_{O F F}$ ratio of $\left(\sim 6 \times 10^{12}\right), I_{a m b}$ as $1 \times 10^{-17}(\mathrm{~A} / \mu m), f_{T}$ of $(\sim$ $\left.28 \mathrm{GH}_{Z}\right)$. Ashita et al. [15] investigated a inverted-C TFET (ICTFET) with SCOPs which provides both lateral and vertical tunneling to boost the ON current. The authors have reported $I_{O N} / I_{O F F}$ ratio of $\left(4.5 \times 10^{9}\right)$, SS of $(48 \mathrm{mV} /$ decade $), f_{T}$ of $\left(1.19 G H_{Z}\right)$. Chandan et al. [16] proposed a metal strip based TFET (MS-ED-TFET) to overcome low switching ratio, SS and analog/RF performance and acheived an $I_{O N} / I_{O F F}$ of $\left(8.92 \times 10^{8}\right)$, SS of $(8.07 \mathrm{mV} /$ decade $), g_{m}$ of 0.007 $\mathrm{mS}, f_{T}$ as $\left(0.17 G H_{Z}\right)$. Shaikh et al. [17] presented a quadruple-gate TFET with drain engineering (DEQG-TFET) and acheived an $I_{O N} / I_{O F F}$ ratio of $(1.79$ $\left.\times 10^{12}\right), f_{T}$ of $\left(\sim 34 G H_{Z}\right), g_{m}$ of $0.261 \mathrm{mS}, \mathrm{GBP}$ of $\left(3.9 \mathrm{GH}_{Z}\right)$. kumar et al. [18] proposed a stack-gate approach TFET with dual material (DMDODG-TFET) to and reported an $I_{O N} / I_{O F F}$ ratio of $\left(5 \times 10^{11}\right)$, SS of ( $18.5 \mathrm{mV} /$ decade $), f_{T}$ of $\left(\sim 8.8 G H_{Z}\right), g_{m}$ of 0.09 $\mathrm{mS}$. Joshi et al. [19] investigated an extended source TFET (ESDG-TFET) and reported an $I_{O N} / I_{O F F}$ of $\left(2.57 \times 10^{12}\right)$, SS of $(12.24 \mathrm{mV} /$ decade $), g_{m}$ of $0.238 \mathrm{mS}$, $f_{T}$ of $\left(37.7 \mathrm{GH}_{Z}\right)$

In this work, we propose DMSGO-DP-TFET to address the ON-current, ambipolarity, and improve the analog/RF, and linearity performance. For this, the combination of $\left(\phi_{3}<\phi_{2}\right)$ workfunction and high-K dielectric pocket at the drain junction leads to an increased tunneling barrier width and reduced lateral electric field. As a result, ambipolar current reduces when negative $V_{G S}$ is applied. Similarly, $\left(\phi_{1}<\phi_{2}\right)$ work function and the low-K dielectric pocket at the source junction reduces the tunneling width $(\lambda)$ and improves the lateral electric field, which results in an improved tunneling current.

This paper is organized as follows: Section 2 presents the structural, simulation details and process flow. Sec- tion 3 presents the structural optimization results. Section 4 describes the DC, analog/RF, and linearity performance. Lastly, Section 5 concludes the main findings of this work.

\section{Device structure, parameters, simulation setup and process flow}

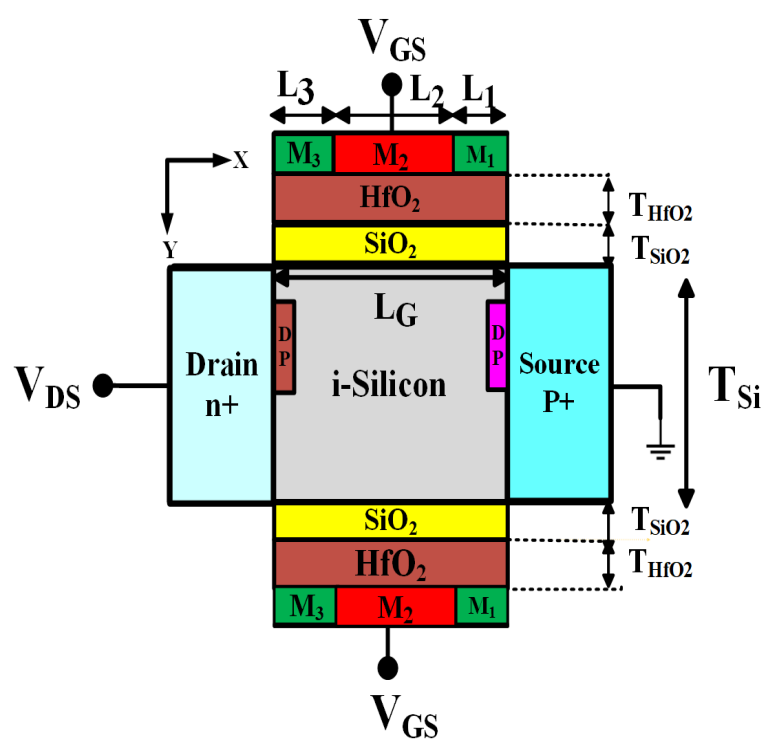

Fig. 1 Cross-sectional view of proposed DMSGO-DP-TFET

The 2D structural view of the proposed DMSGODP-TFET for the parameters listed in Table 1 is illustrated in Fig. 1. The length of the stack gate $\left(\mathrm{SiO}_{2}+\mathrm{HfO}_{2}\right)$ is considered $50 \mathrm{~nm}$ with $\mathrm{SiO}_{2}$ layer thickness of $(0.8$ $\mathrm{nm})$ and $\mathrm{HfO}_{2}$ oxide layer thickness of (1.2 nm) [18]. Further, the entire gate is split into three segments labelled as tunneling gate $\left(M_{1}\right)$, control gate $\left(M_{2}\right)$ and auxiliary gate $\left(M_{3}\right)$ with lengths $\left(L_{1}, L_{2}, L_{3}\right)$ and work functions $\left(\phi_{1}, \phi_{2}, \phi_{3}\right)$ respectively. In the case of singlematerial stack gate oxide-dielectric pocket-TFET (SMSGODP-TFET) the gate work functions $\left(\phi_{1}=\phi_{2}=\phi_{3}\right)$ is considered. The control gate workfunction $\left(\phi_{2}\right)$ is considered $4.4 \mathrm{eV}$ corresponding to the metal molybdenum (Mo) $(4.36 \mathrm{eV}-4.95 \mathrm{eV})$. The work functions $\left(\phi_{1}\right.$ and $\left.\phi_{3}\right)$ are considered $4.0 \mathrm{eV}$ corresponding to metal aluminum (Al) $(4.0 \mathrm{eV}-4.26 \mathrm{eV})$ [20]. In DMSGOTFET without dielectric pocket $I_{O N} / I_{O F F}$ is noted as $2.25 \times 10^{11}$. A low- $\mathrm{K}$ dielectric pocket is considered at the source-channel interface to increase the $I_{O N}$. Whereas, high-K dielectric pocket at the drain-channel interface to lower the $I_{O F F}$. Due to this, $I_{O N} / I_{O F F}$ is 
Table 1 Design parameters used in device simulation

\begin{tabular}{lcc}
\hline Parameters & DMCG-DP-TFET & DMSGO-DP-TFET \\
\hline Gate Length $\left(\mathrm{L}_{G}\right)$ & $50 \mathrm{~nm}$ & $50 \mathrm{~nm}$ \\
$\mathrm{SiO}_{2}$ thickness $\left(\mathrm{T}_{\mathrm{SiO}_{2}}\right)$ & $2 \mathrm{~nm}$ & $0.8 \mathrm{~nm}$ \\
$\mathrm{HfO}_{2}$ thickness $\left(\mathrm{T}_{\mathrm{H} f \mathrm{O}_{2}}\right)$ & - & $1.2 \mathrm{~nm}$ \\
Pocket thickness & $2 \mathrm{~nm}$ & $2 \mathrm{~nm}$ \\
Pocket height & $4 \mathrm{~nm}$ & $4 \mathrm{~nm}$ \\
Silicon film thickness $\left(\mathrm{T}_{s i}\right)$ & $10 \mathrm{~nm}$ & $10 \mathrm{~nm}$ \\
Channel doping $\left(\mathrm{N}_{c h}\right)$ & $1 \times 10^{17} \mathrm{~cm}^{-3}$ & $1 \times 10^{17} \mathrm{~cm}^{-3}$ \\
Source doping $\left(\mathrm{p}\right.$ type) $\left(\mathrm{N}_{S}\right)$ & $1 \times 10^{20} \mathrm{~cm}^{-3}$ & $1 \times 10^{20} \mathrm{~cm}^{-3}$ \\
Drain doping(n type) $\left(\mathrm{N}_{D}\right)$ & $5 \times 10^{18} \mathrm{~cm}^{-3}$ & $5 \times 10^{18} \mathrm{~cm}^{-3}$ \\
$\mathrm{HfO}_{2}$ dielectric constant $(\mathrm{K})$ & 25 & 25 \\
Tunneling gate Length $\left(\mathrm{L}_{1}\right)$ & $10 \mathrm{~nm}$ & $10 \mathrm{~nm}$ \\
Control gate Length $\left(\mathrm{L}_{2}\right)$ & $30 \mathrm{~nm}$ & $30 \mathrm{~nm}$ \\
Auxiliary gate Length $\left(\mathrm{L}_{3}\right)$ & $10 \mathrm{~nm}$ & $10 \mathrm{~nm}$ \\
Tunnel gate workfunction $\left(\phi_{1}\right)$ & $4.0 \mathrm{eV}$ & $4.0 \mathrm{eV}$ \\
Control gate workfunction $\left(\phi_{2}\right)$ & $4.4 \mathrm{eV}$ & $4.4 \mathrm{eV}$ \\
Auxiliary gate workfunction $\left(\phi_{3}\right)$ & $4.0 \mathrm{eV}$ & $4.0 \mathrm{eV}$ \\
\hline
\end{tabular}

noted as $2.83 \times 10^{12}$ in the proposed device using Atlas Silvaco TCAD simulations.

In our simulation, nonlocal band-to-band tunneling (BTBT) model, which uses the Wentzel-KramersBrillouin method, is considered to measure the tunneling probability across the junction. For this, the quantum tunneling regions are defined at the source/channel interface to account for carrier tunneling in the $\mathrm{ON}$ state, whereas at the drain/channel interface to account for ambipolar behaviour of the device. Shockley-ReadHall model is enabled to consider minority carrier recombination effects for numerical solutions. The bandgap narrowing method (BGN) is used due to heavily doped concentrations in source and drain regions. FLDMOB mobility model is used to consider the velocity saturation effects because of the lateral electrical field. The simulation study carried out in this work uses a nonlocal BTBT model calibrated with the data reported in [8], which was previously calibrated, with the experimental data obtained from the fabricated device [21]. Fig. 2 shows the comparison of results reported in [8] and the proposed device using TCAD.

The proposed device can be fabricated using the similar method reported in [19, 26]. The conceptual process flow of the proposed device is illustrated in Fig. 3(a)-(j). Firstly, a p-i-n structure can be formed using epitaxial growth process as shown in Fig. 3(a) and Fig. 3(b) shows the deposition of high-K and low$\mathrm{K}$ dielectrics at the drain and source junctions. In the next step, etching is done to form the dielectric pockets as shown in Fig. 3(c). Then, $\mathrm{SiO}_{2}$ can be grown on the p-i-n structure by the oxidation process as illustrated in Fig. 3(d). Atomic layer deposition (ALD) is used to deposit a thin $\mathrm{HfO}_{2}$ film as illustrated in Fig. 3(e). In the next- step, as shown in Fig. 3(f), cre-

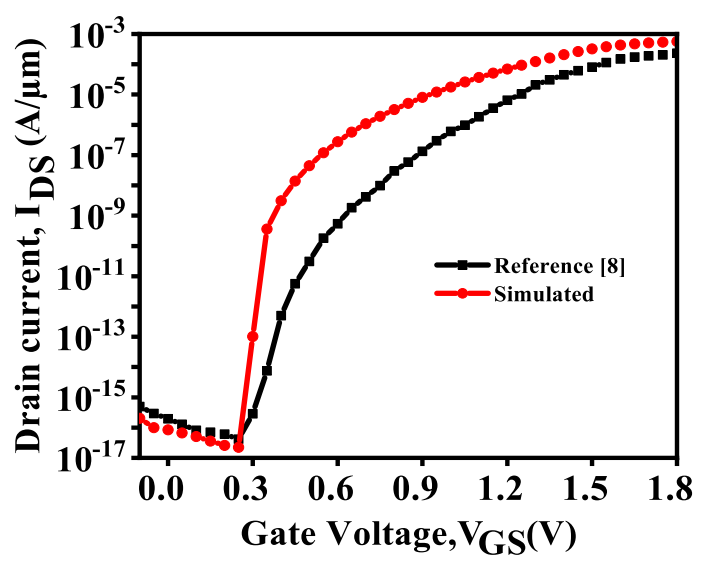

Fig. 2 Comparison of conventional n-TFET [8] and DMSGO-DP-TFET $I_{D S}-V_{G S}$ characteristics at $V_{D S}=1.0$ $\mathrm{V}$

ation of the control gate can be done using masking and metallization of molybdenum (Mo) material. Moreover, auxiliary and tunnel gates can also metalized with aluminium (Al) material using the self-aligned symmetrical spacer method as illustrated in Fig. 3(g). Next, selective etching can be done for electrode formation as illustrated in Fig. 3(h). Similarly, the back gate formation can be done as illustrated in Fig. 3(i). Finally, Fig. $3(\mathrm{j})$ presents the metallization and patterning of source and drain contacts using Mo material as it provides the workfunction ranges from $4.36 \mathrm{eV}$ to $4.95 \mathrm{eV}$ 
(a)

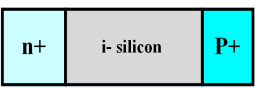

(b)

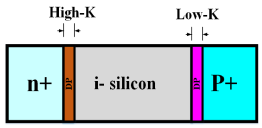

(c)

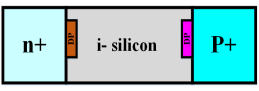

(d)

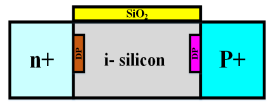

(e)
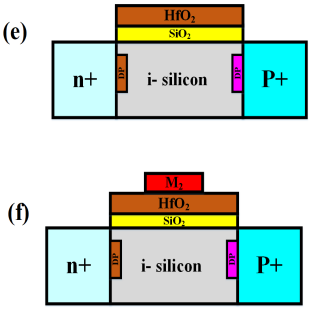

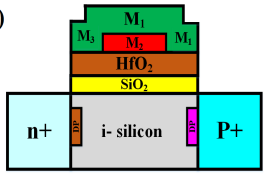

(h)

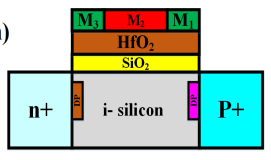

(i)

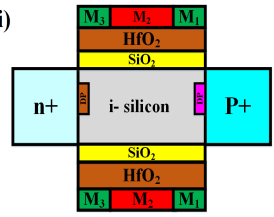

(j)

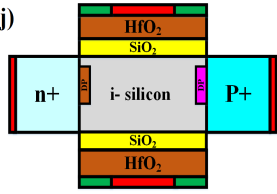

Fig. 3 Fabrication process flow for the proposed DMSGODP-TFET structure

\section{Results and discussion}

\subsection{Workfunction optimization Results}

To align the band structure at the source and drain junctions and to modulate the carriers through the channel, the entire gate length of DMSGO-DP-TFET is splitted into three segments known as tunneling gate $\left(M_{1}\right)$, control gate $\left(M_{2}\right)$, and auxiliary gate $\left(M_{3}\right)$ with workfunctions $\phi_{1}, \phi_{2}$ and $\phi_{3}$, respectively as illustrated in Fig. 1. By fixing $\left(\phi_{1}\right.$ and $\left.\phi_{3}\right)$ and varying $\left(\phi_{2}\right)$, better switching ratio and minimum ambipolar current is observed for the combination $\left(\phi_{1}=\phi_{3}<\phi_{2}\right)$ as illustrated in Fig. 4(a) and table 2. Fig. 4(b) and Fig. 4(c) shows ON-state and the OFF-state band diagrams for the proposed DMSGO-DP-TFET by varying $\left(\phi_{2}\right)$ and fixing $\left(\phi_{1}=\phi_{3}=4.0 \mathrm{eV}\right)$. From Fig. $4(\mathrm{c})$, it can be seen that tunneling width increases with an increase in $\left(\phi_{2}\right)$. This results in band overlap reduction on the source side and decreases the tunneling current. Whereas, the band overlap has been noted at the drain junction for $\left(\phi_{2}>4.4 \mathrm{eV}\right)$ and tunneling is observed in the OFF state, also $\left(\phi_{2}\right)$ work function variation in the ON-state does not shows a significant change in the tunneling current until the workfunction $\left(\phi_{2}=4.4 \mathrm{eV}\right)$. Also, as shown in Fig. 4(c), a decrease in band overlap is seen for

$\left(\phi_{2}>4.4 \mathrm{eV}\right)$, which results in a decrease in ON-state current $\left(I_{O N}\right)$

\subsection{Dielectric pocket optimization results}

To further improve $I_{O N}$ and minimize the ambipolar current, optimized low-K and high -K dielectric pockets are inserted at the source and drain junctions, respectively. Fig. 5(a) shows the comparative $I_{D S}-V_{G S}$ characteristics for different dielectric materials at the source and drain junctions in DMSGO-DP-TFET. Results demonstrate that combination of low-K dielectric pocket (air) at the source junction and high-K dielectric pocket $\left(\mathrm{HfO}_{2}\right)$ at the drain junction shows higher $I_{O N}$ and minimum ambipolar current because of tunneling width and band alignment at the source and drain junctions. Fig. 5(b) shows $I_{D S}-V_{G S}$ characteristics of DMSGO-DP-TFET for various dielectric pocket heights at the source junction by fixing the dielectric pocket height on the drain side. From the Results, it has been noted that, dielectric pocket of $4 \mathrm{~nm}$ height at the source junction shows better $I_{O N}$ because of increased electric field and decrease in tunneling width. Similarly, as shown in Fig. 5(c), fixing the height of the low$\mathrm{K}$ dielectric pocket and varying the high-K dielectric pocket, it has observed that at the drain junction, dielectric pocket of $4 \mathrm{~nm}$ height shows minimum ambipolar current with better switching ratio $\left(I_{O N} / I_{O F F}\right)$. Fig. $5(\mathrm{~d})$ depicts the comparative $I_{D S^{-}} V_{G S}$ characteristics of DMSGO-DP-TFET for different pocket thickness. From the same figure, it can be noted that pocket thickness of $2 \mathrm{~nm}$ at the tunneling junction shows better tunneling current $I_{O N}$ and minimum ambipolar current due to the band alignments at the source and drain junctions, respectively.

\subsection{Gate length optimization}

In this section, the gate segments of the proposed device is optimized. In this regard, Tables $3-5$ shows the variations in $I_{O N}, I_{O F F}, I_{a m b}, I_{O N} / I_{O F F}$ for various combinations of $L_{1}, L_{2}$ and $L_{3}$. The optimization of $L_{3}$ at constant $L_{1}=10 \mathrm{~nm}$ is shown in Table 3. Further, the optimization of $L_{1}$ at constant $L_{3}=15 \mathrm{~nm}$ is shown in Table 4 . Table 5 shows the $L_{1}$ and $L_{3}$ variations at $L_{1}=L_{3}$. From this analysis, it has been noted that $L_{3}=15 \mathrm{~nm}$ performs better in terms of $I_{O N}, I_{O F F}$, $I_{a m b}$. However, $L_{1}=L_{3}=10 \mathrm{~nm}$ shows better results in terms of $I_{O N} / I_{O F F}$ and is considered as optimized gate lengths in this work.

For the optimized dielectric pockets illustrated in Fig. 1, based on $\left(\phi_{1}, \phi_{2}, \phi_{3}\right)$, various device structures 


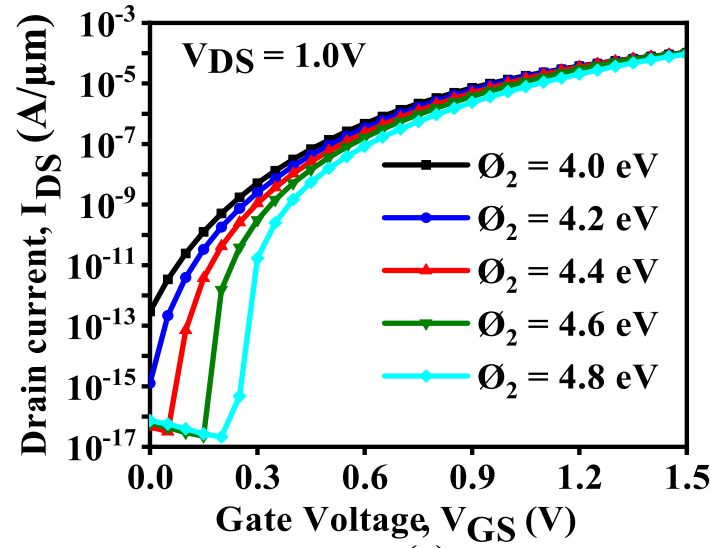

(a)

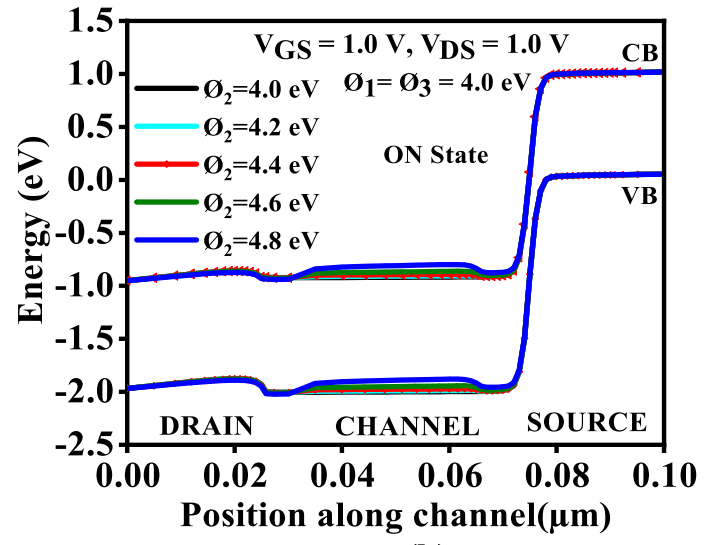

(b)

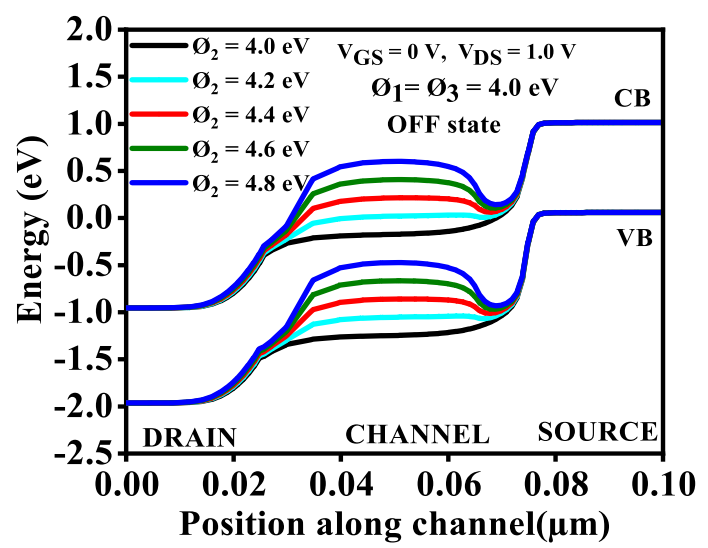

(c)

Fig. 4 Comparative results of (a) workfunction optimization (b) ON state band variation with $\left(\phi_{2}\right)$ (c) OFF state band variation with $\left(\phi_{2}\right)$ for the proposed device DMSGO-DP-TFET

Table 2 Impact of $\phi_{2}$ on $I_{O N} / I_{O F F}$ with $\left(\phi_{1}=\phi_{3}\right)$

\begin{tabular}{lllll}
\hline$\phi_{2}$ & $I_{O N}(\mathrm{~A} / \mu m)$ & $I_{O F F}(\mathrm{~A} / \mu m)$ & $I_{O N} / I_{O F F}$ & $I_{a m b}(\mathrm{~A} / \mu m)$ \\
\hline $4.0 \mathrm{eV}$ & $1.36 \times 10^{-5}$ & $2.96 \times 10^{-13}$ & $4.58 \times 10^{7}$ & $3.58 \times 10^{-16}$ \\
$4.2 \mathrm{eV}$ & $1.23 \times 10^{-5}$ & $1.26 \times 10^{-15}$ & $9.79 \times 10^{9}$ & $3.70 \times 10^{-16}$ \\
$4.4 \mathrm{eV}$ & $1.02 \times 10^{-5}$ & $4.58 \times 10^{-17}$ & $2.23 \times 10^{11}$ & $3.76 \times 10^{-16}$ \\
$4.6 \mathrm{eV}$ & $8.11 \times 10^{-6}$ & $6.18 \times 10^{-17}$ & $1.31 \times 10^{11}$ & $3.79 \times 10^{-16}$ \\
$4.8 \mathrm{eV}$ & $5.36 \times 10^{-6}$ & $7.31 \times 10^{-17}$ & $7.33 \times 10^{10}$ & $3.80 \times 10^{-16}$ \\
\hline
\end{tabular}

Table $3 L_{3}$ Gate segment optimization at constant $L_{1}=10 \mathrm{~nm}$

\begin{tabular}{lllll}
\hline$L_{3}$ & $I_{O N}(\mathrm{~A} / \mu m)$ & $I_{O F F}(\mathrm{~A} / \mu m)$ & $I_{O N} / I_{O F F}$ & $I_{a m b}(\mathrm{~A} / \mu m)$ \\
\hline $5 \mathrm{~nm}$ & $1.51 \times 10^{-4}$ & $3.16 \times 10^{-14}$ & $4.78 \times 10^{9}$ & $4.09 \times 10^{-13}$ \\
$10 \mathrm{~nm}$ & $1.48 \times 10^{-4}$ & $5.23 \times 10^{-17}$ & $2.83 \times 10^{12}$ & $5.21 \times 10^{-16}$ \\
$15 \mathrm{~nm}$ & $1.59 \times 10^{-4}$ & $4.36 \times 10^{-14}$ & $3.65 \times 10^{9}$ & $3.65 \times 10^{-16}$ \\
$20 \mathrm{~nm}$ & $1.62 \times 10^{-4}$ & $6.21 \times 10^{-14}$ & $2.61 \times 10^{9}$ & $3.64 \times 10^{-16}$ \\
\hline
\end{tabular}

Table $4 L_{1}$ Gate segment optimization at constant $L_{3}=15 \mathrm{~nm}$

\begin{tabular}{lllll}
\hline$L_{1}$ & $I_{O N}(\mathrm{~A} / \mu m)$ & $I_{O F F}(\mathrm{~A} / \mu m)$ & $I_{O N} / I_{O F F}$ & $I_{a m b}(\mathrm{~A} / \mu m)$ \\
\hline $5 \mathrm{~nm}$ & $8.51 \times 10^{-5}$ & $3.24 \times 10^{-17}$ & $2.63 \times 10^{12}$ & $3.66 \times 10^{-16}$ \\
$10 \mathrm{~nm}$ & $1.59 \times 10^{-4}$ & $4.36 \times 10^{-14}$ & $3.65 \times 10^{9}$ & $3.66 \times 10^{-16}$ \\
$15 \mathrm{~nm}$ & $1.79 \times 10^{-4}$ & $1.86 \times 10^{-11}$ & $9.62 \times 10^{6}$ & $3.66 \times 10^{-16}$ \\
$20 \mathrm{~nm}$ & $1.88 \times 10^{-4}$ & $3.8 \times 10^{-11}$ & $4.96 \times 10^{6}$ & $3.66 \times 10^{-16}$ \\
\hline
\end{tabular}




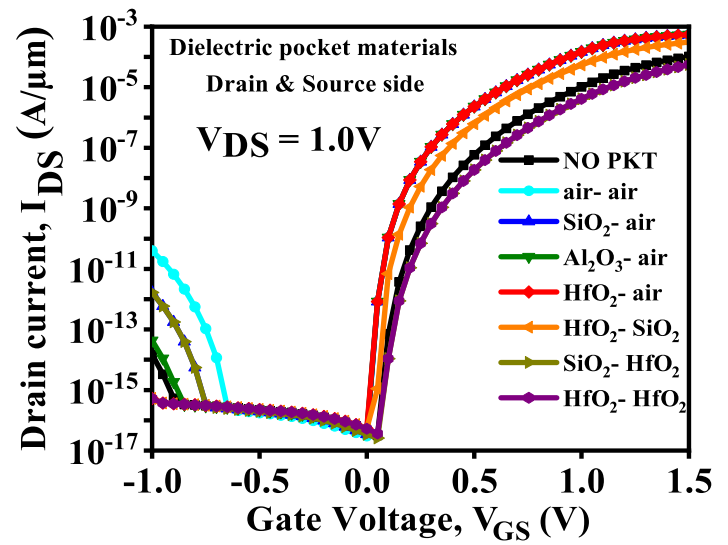

(a)

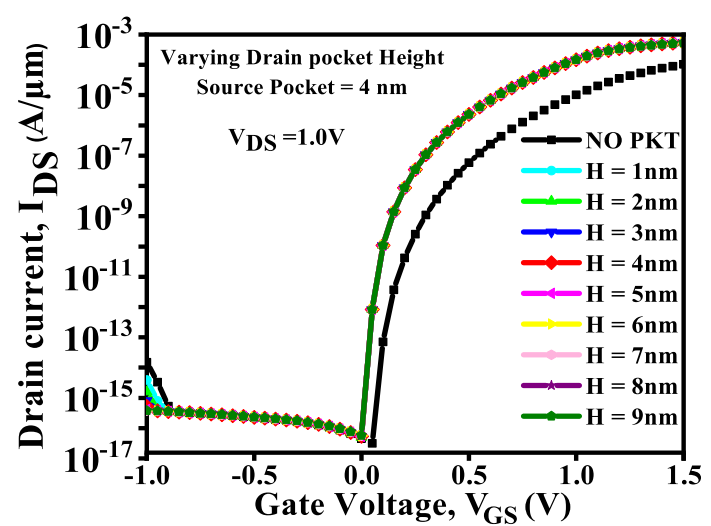

(c)

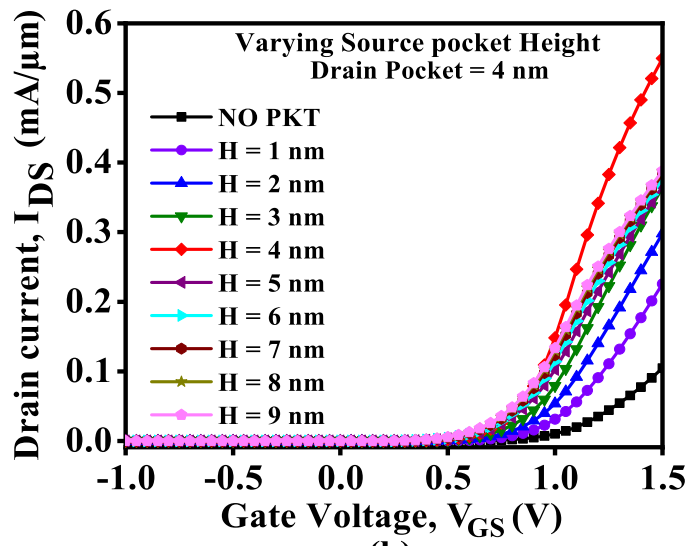

(b)

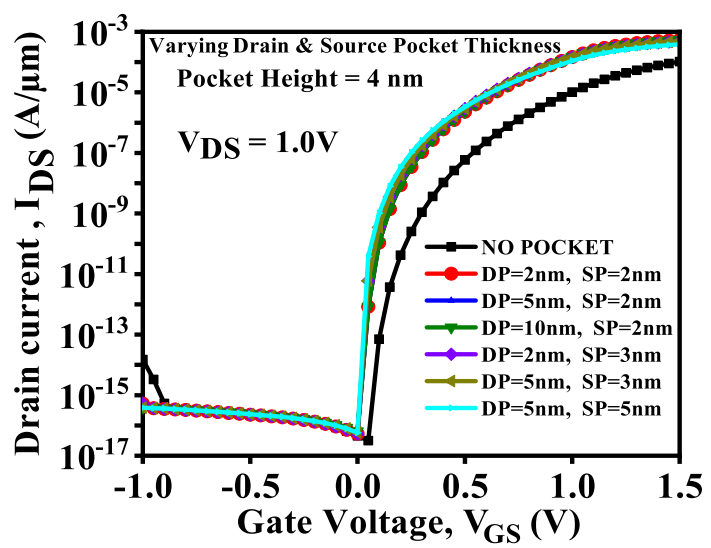

(d)

Fig. 5 Comparative optimization results for (a) Dielectric materials (b) Low-K dielectric pocket height (c) high-K dielectric pocket height (d) Dielectric pocket thickness by fixing heights in proposed DMSGO-DP-TFET.

Table $5 L_{1}$ and $L_{3}$ Gate segments optimization with $L_{1}=L_{3}$

\begin{tabular}{lllll}
\hline$L_{1}$ & $I_{O N}(\mathrm{~A} / \mu m)$ & $I_{O F F}(\mathrm{~A} / \mu m)$ & $I_{O N} / I_{O F F}$ & $I_{a m b}(\mathrm{~A} / \mu m)$ \\
\hline $5 \mathrm{~nm}$ & $8.45 \times 10^{-5}$ & $9.01 \times 10^{-17}$ & $9.38 \times 10^{11}$ & $4.09 \times 10^{-13}$ \\
$10 \mathrm{~nm}$ & $1.48 \times 10^{-4}$ & $5.23 \times 10^{-17}$ & $2.83 \times 10^{12}$ & $5.21 \times 10^{-16}$ \\
$15 \mathrm{~nm}$ & $1.79 \times 10^{-4}$ & $1.86 \times 10^{-11}$ & $9.62 \times 10^{6}$ & $3.66 \times 10^{-16}$ \\
$20 \mathrm{~nm}$ & $1.97 \times 10^{-4}$ & $4.0 \times 10^{-11}$ & $4.91 \times 10^{6}$ & $4.91 \times 10^{-16}$ \\
\hline
\end{tabular}

Table 6 Possible devices with different work functions

\begin{tabular}{llll}
\hline Possible device & $\phi_{1}$ & $\phi_{2}$ & $\phi_{3}$ \\
\hline SMSGO-DP-TFET & $4.4 \mathrm{eV}$ & $4.4 \mathrm{eV}$ & $4.4 \mathrm{eV}$ \\
DMSGO-DP-TFET1 & $4.0 \mathrm{eV}$ & $4.4 \mathrm{eV}$ & $4.4 \mathrm{eV}$ \\
DMSGO-DP-TFET2 & $4.4 \mathrm{eV}$ & $4.4 \mathrm{eV}$ & $4.0 \mathrm{eV}$ \\
DMSGO-DP-TFET & $4.0 \mathrm{eV}$ & $4.4 \mathrm{eV}$ & $4.0 \mathrm{eV}$ \\
\hline
\end{tabular}

can be formed as presented in Table 6. Further, a comparative analysis among these devices is done in terms of their carrier concentration, band diagrams, tunneling rate, surface potential, electric field variation, and $I_{D S^{-}}$ $V_{G S}$ characteristics. For SMSGO-TFET, all the three workfunctions $\left(\phi_{1}, \phi_{2}, \phi_{3}\right)$ of the gate material has been considered equal (4.4 eV). Fig. 6(a) depicts the carrier concentration variation with distance for SMSGO-DP-
TFET, DMSGO-DP-TFET1, DMSGO-DP-TFET2 and DMSGO-DP-TFET. From the figure, higher electron concentration is observed for DMSGO-DP-TFET due to decrease in tunneling width as illustrated in Fig. 6 (b). Therefore, better band-to band tunneling rate is observed for DMSGO-DP-TFET as shown in Fig. 6(c). Further, large increase in surface potential and electric field is observed at the source junction for DMSGO-DP- 


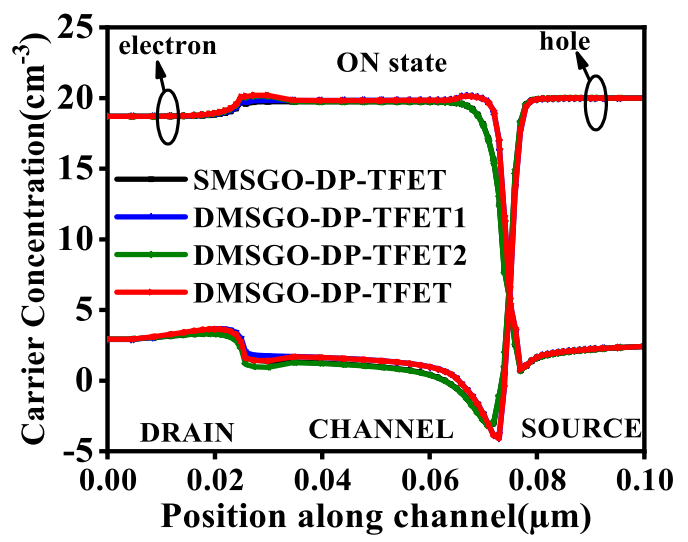

(a)

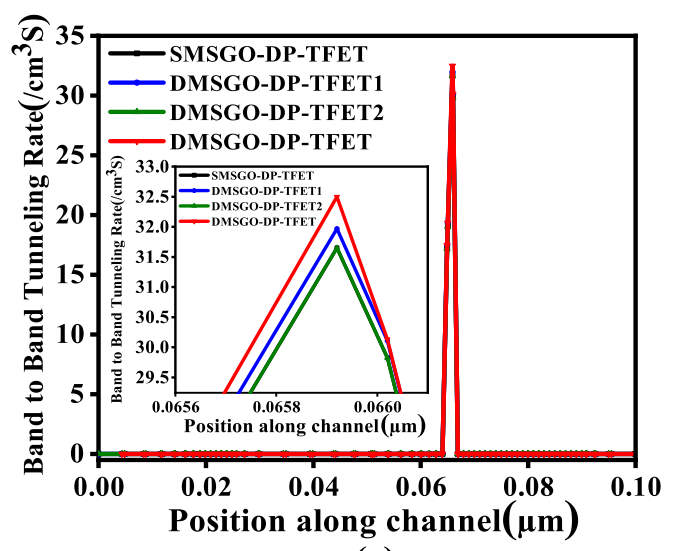

(c)

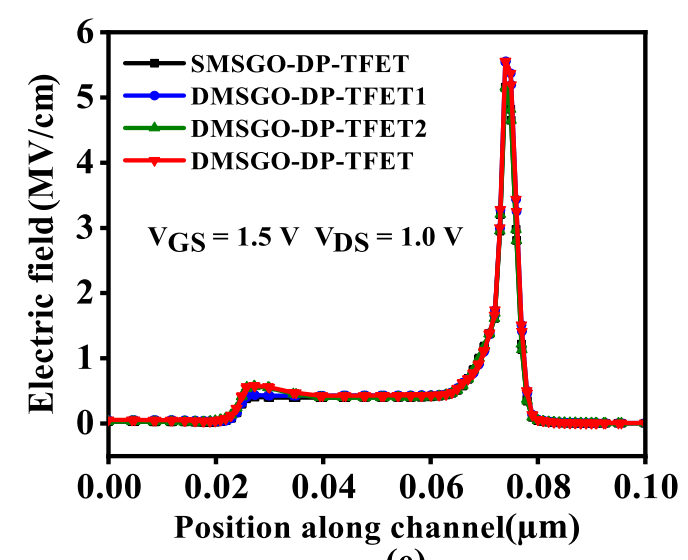

(e)

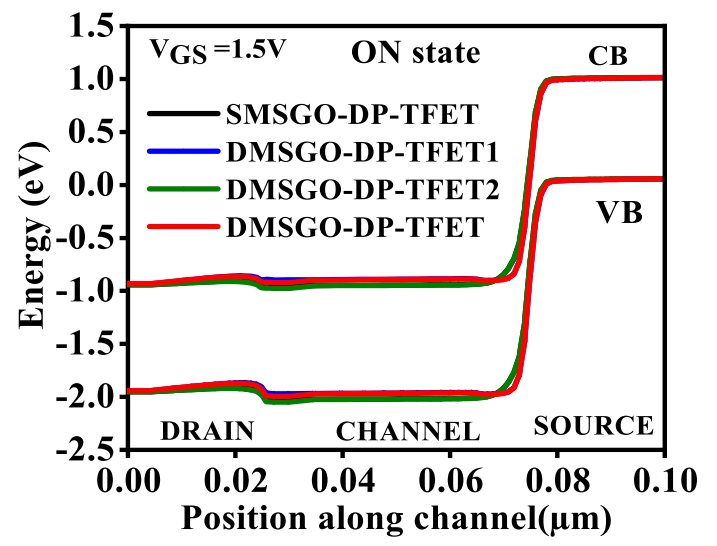

(b)

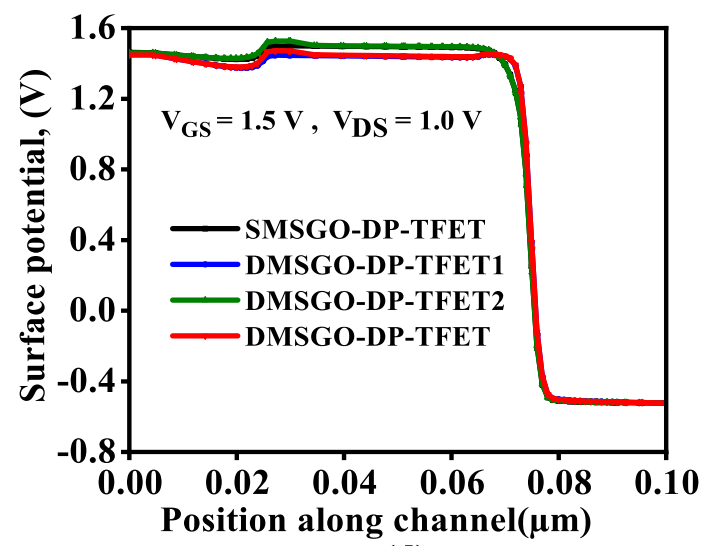

(d)

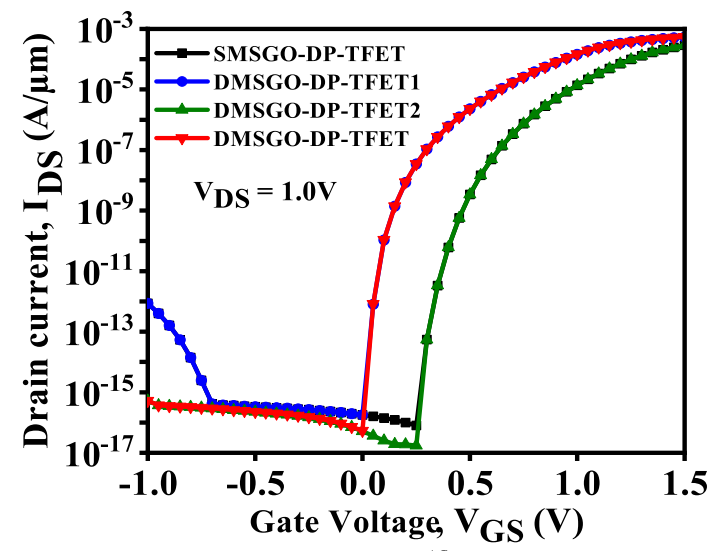

(f)

Fig. 6 Comparative results of (a) Carrier concentration variation (b) Band variation (c) Tunneling rate (d) surface potential variation (e) Electric field variation (f) $I_{D S}-V_{G S}$ characteristics for SMSGO-DP-TFET, DMSGO-DP-TFET1, DMSGO-DPTFET2 and DMSGO-DP-TFET at $\left(V_{D S}=1.0 \mathrm{~V}, V_{G S}=1.5 \mathrm{~V}\right)$

TFET as illustrated in Fig. 6(d) and Fig. 6(e), respectively. Thus, maximum tunneling occurs at the source junction, as the BTBT rate $\left(G_{B T B T}\right)$ at the tunneling junction depends on the electric field $(\varepsilon)$ leads to an increase in BTBT rate as per the expression [22].

$G_{B T B T}=A \varepsilon^{\sigma} \exp \left(-\frac{B}{\varepsilon}\right)$
Where A, B are constants which are related to the electron's effective mass and the tunnelling probability, $\varepsilon$ is the electric field and $\sigma$ is the transition constant. Further, the probability of tunneling of the car- 


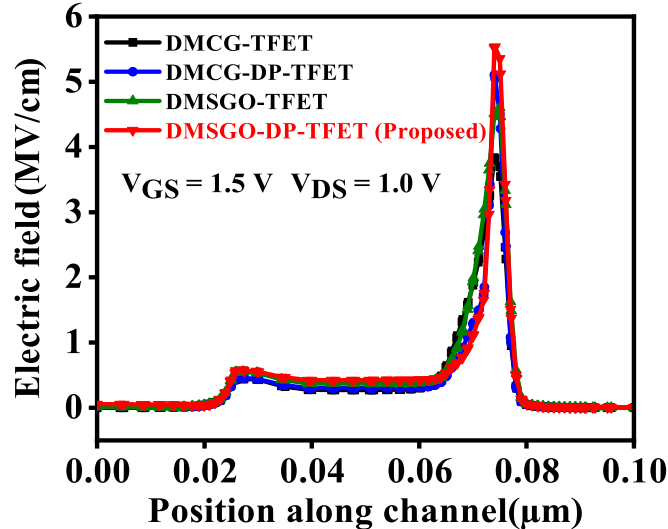

(a)

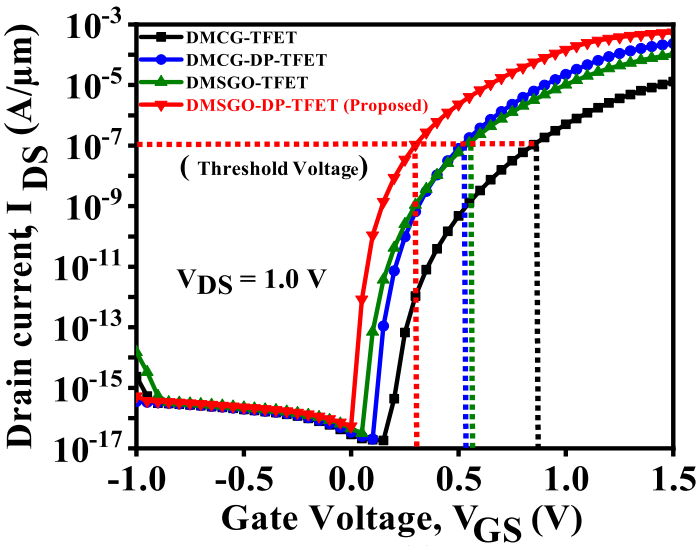

(c)

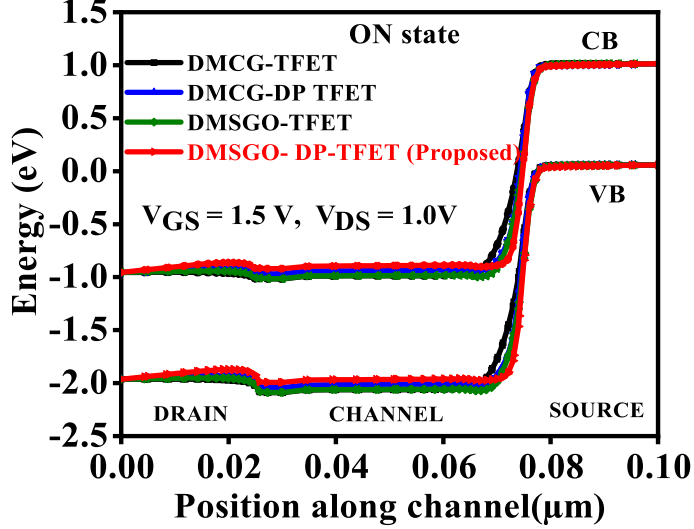

(b)

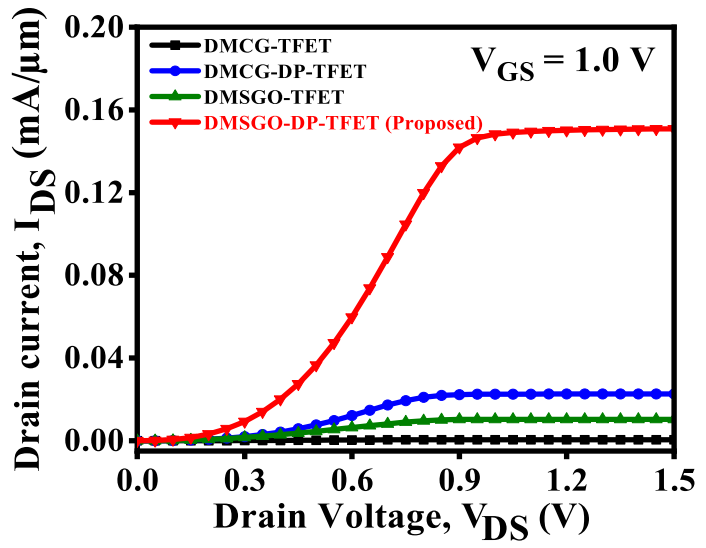

(d)

Fig. 7 Comparative results of (a) Electric field variation (b) Band variation (c) $I_{D S}-V_{G S}$ characteristics (d) $I_{D S}-V_{D S}$ characteristics for DMCG-TFET, DMCG-DP-TFET, DMSGO-TFET and DMSGO-DP-TFET at $\left(V_{D S}=1.0 \mathrm{~V}, V_{G S}=1.5 \mathrm{~V}\right)$

rier $\left(T_{W K B}\right)$ at the tunneling barrier is analyzed by the approximation of Wentzel-Kramer-Brillouin (WKB) [23].

$T_{W K B} \propto \exp \left[-\frac{4 \lambda \sqrt{2 m^{*}} \sqrt{E_{g}{ }^{3}}}{3 q h\left(E_{g}+\Delta \phi\right)}\right]$

Where $m^{*}$ is the effective mass of an electron, q represents the electron charge, $\mathrm{h}$ represents Plank's constant, $E_{g}$ is the effective bandgap, $\lambda$ is the width of tunneling barrier, and $\Delta \phi$ represents the energy overlap where the tunneling occurs. Therefore, as shown in Fig. 6(f), a significant increase in tunneling current is observed. Fig. 6 and Table 7 show that DMSGO-DPTFET performs better than other device structures.

\section{$4 \mathrm{DC}$, analog/RF and linearity performance}

\subsection{DC characteristics}

In this section, comparative DC characteristics of DMCGTFET, DMCG-DP-TFET, DMSGO-TFET and DMSGODP-TFET has been analyzed. In this regard, the elec- tric field variation with distance for the above device structures are illustrated in Fig. 7(a). From the simulation results, a higher electric field at the sourcechannel interface is noted for DMSGO-DP-TFET, as a result, minimum tunneling barrier width is noted for DMSGO-DP-TFET as illustrated in Fig. 7(b). Further, Fig. $7(\mathrm{c})$ illustrates the comparative $I_{D S^{-}} V_{G S}$ characteristics variation of DMCG-TFET, DMCG-DP-TFET, DMSGO-TFET and DMSGO-DP-TFET. From the figure, a significant increase in ON-state current is observed for DMSGO-DP-TFET because BTBT depends on the electric field and tunneling width at the source junction [22]. Fig. 7(d) depicts the comparative output characteristics for various device structures at $V_{G S}=1$ V. From the figure, it can be noted that the tunneling current $I_{D S}$ rises at first as $V_{D S}$ increases from 0 to $0.8 \mathrm{~V}$, then saturates because of velocity saturation as $V_{D S}$ increases beyond $0.8 \mathrm{~V}$. The tunneling current does not change significantly from $V_{D S}=0.8 \mathrm{~V}$ onwards, so the current remains constant. Further, noted that due to employment of dielectric pockets at the source and drain junctions, reduced $V_{T}$, improved ON-state 


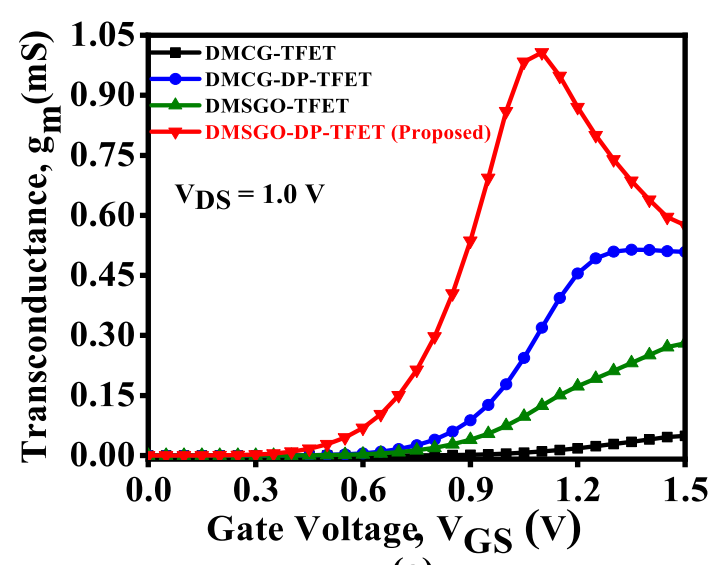

(a)

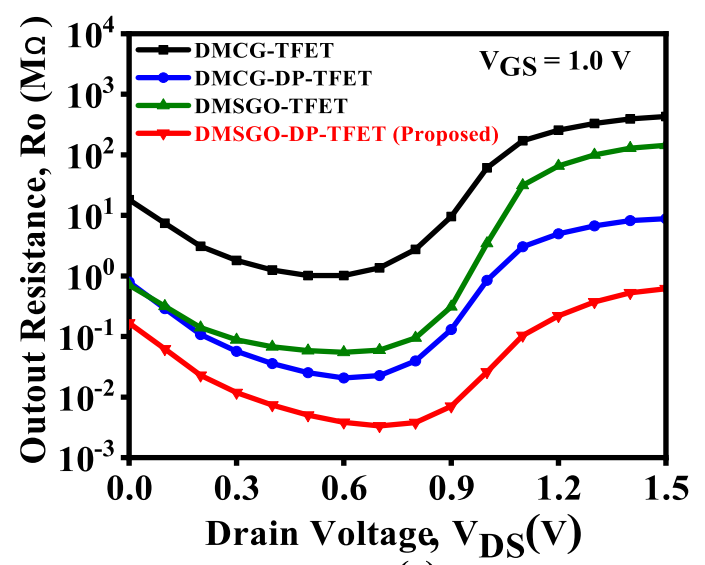

(c)

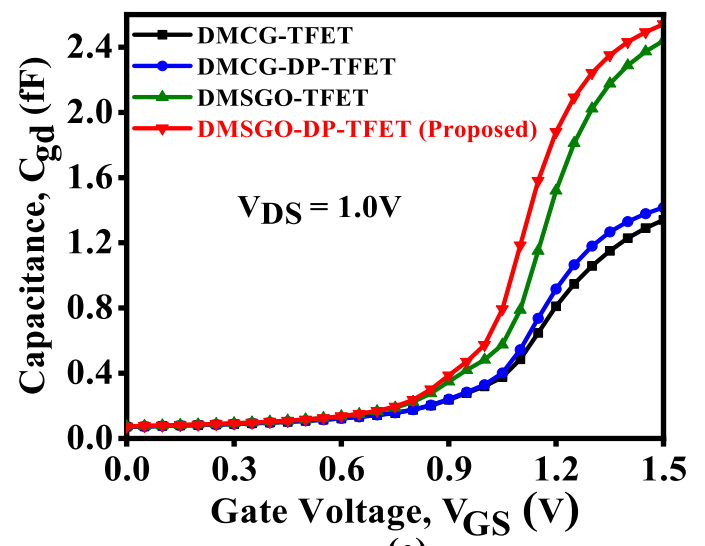

(e)

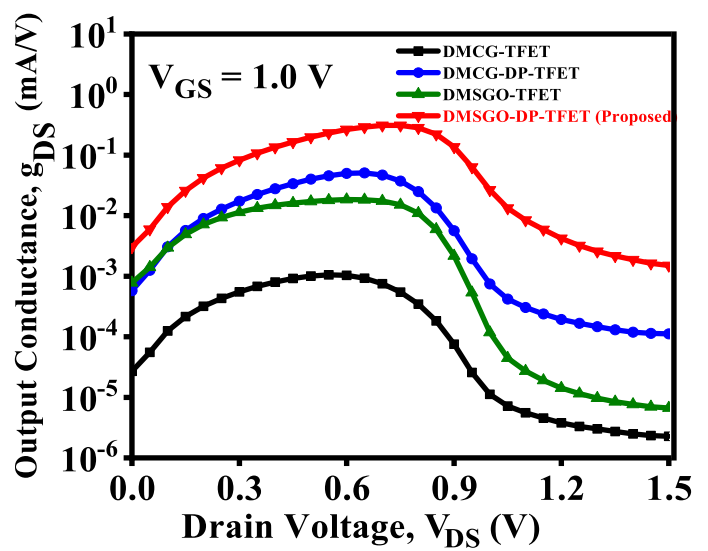

(b)

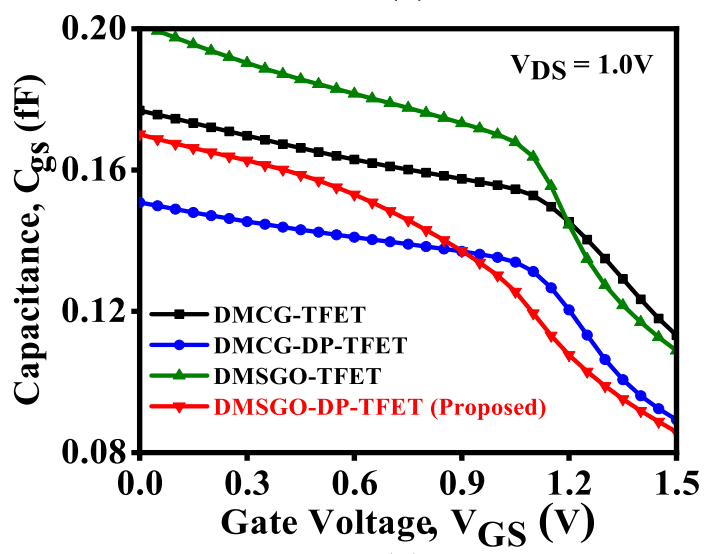

(d)

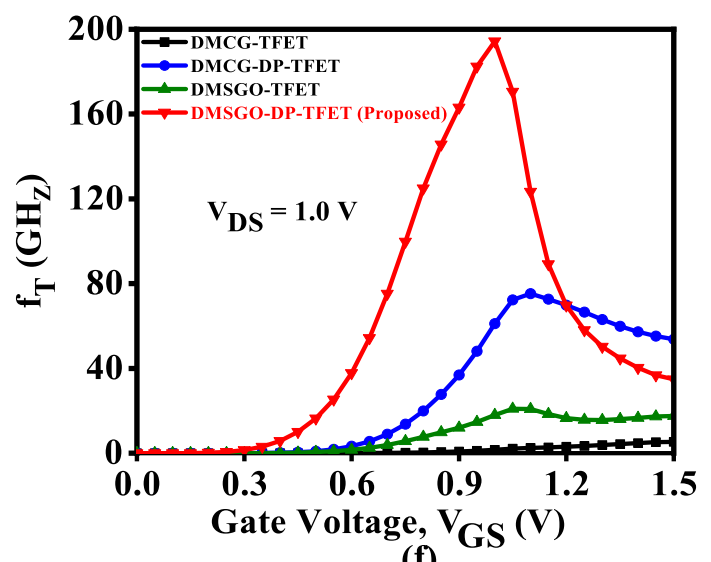

(f)

Fig. 8 Comparative performance of (a) $g_{m}$ (b) $g_{D S}$ (c) $R_{O}$ (d) $C_{g s}$ (e) $C_{g d}$ (f) $f_{T}$ in DMCG-TFET, DMCG-DP-TFET, DMSGO-TFET and DMSGO-DP-TFET

current, reduced ambipolar current, steep subthreshold slope $(\mathrm{SS})$ and a higher switching ratio $\left(2.83 \times 10^{12}\right)$ was observed for DMSGO-DP-TFET compared with other structures as illustrated in Fig. 7 and Table 8.

\subsection{Analog/RF figure of merits}

This section presents performance analysis of DMCGTFET, DMCG-DP-TFET, DMSGO-TFET and DMSGODP-TFET in terms of various analog/RF figure of merits (FOMs) such as $g_{m}, g_{D S}, R_{o}, C_{g s}, C_{g d}, f_{T}$, TGF, $f_{\text {max }}$, TFP, GBP and transit time. Transconductance $\left(g_{m}\right)$ is one of the critical parameters in the analog/RF 
Table 7 Performance comparison of sigle material and dual material TFET with dielectric pockets

\begin{tabular}{lllll}
\hline Parameters & SMSGO-DP-TFET & DMSGO-DP-TFET1 & DMSGO-DP-TFET2 & DMSGO-DP-TFET \\
\hline$I_{O N}(\mathrm{~A} / \mu \mathrm{m})$ & $1.37 \times 10^{-5}$ & $1.41 \times 10^{-4}$ & $1.35 \times 10^{-5}$ & $1.48 \times 10^{-4}$ \\
$I_{O F F}(\mathrm{~A} / \mu \mathrm{m})$ & $1.78 \times 10^{-16}$ & $1.788 \times 10^{-16}$ & $5.18 \times 10^{-17}$ & $5.22 \times 10^{-17}$ \\
$I_{O N} / I_{O F F}$ & $7.68 \times 10^{10}$ & $7.91 \times 10^{11}$ & $2.61 \times 10^{11}$ & $2.83 \times 10^{12}$ \\
$V_{T H}(\mathrm{~V})$ & 0.63 & 0.29 & 0.63 & 0.29 \\
$S S(m V /$ dec. $)$ & 32.7 & 17.3 & 28.7 & 15.8 \\
Electric field $(\mathrm{MV} / \mathrm{cm})$ & 5.15 & 5.55 & 5.16 & 5.56 \\
$g_{m}(s)$ & $8.19 \times 10^{-4}$ & $9.79 \times 10^{-4}$ & $8.27 \times 10^{-4}$ & $1.01 \times 10^{-3}$ \\
\hline
\end{tabular}

and linearity performance analysis of the device. Higher $g_{m}$ results in the higher gain of the device and plays a critical role in attaining higher values of $f_{T}$ and GBP in designing analog circuits [25]. In this regard, Fig. 8 (a) depicts the comparative plots of $g_{m}$ variation with $V_{G S}$ at $V_{D S}=1 \mathrm{~V}$. From the figure, it has been noted that in the subthreshold region $g_{m}$ of all the devices are very small, and it starts increasing due to increase in the ON-state current. Moreover, it starts decreasing after a particular value of $V_{G S}$ due to mobility degradation [24]. Results also show that DMSGO-DP-TFET exhibits higher $g_{m}$ as compared to other device structures.

The next analog/RF performance parameter considered for analysis is output conductance $\left(g_{D S}\right)$. A device with higher $\left(g_{D S}\right)$ is preferred as it provides higher intrinsic gain. In this regard, Fig. 8(b) depicts the variation of $g_{D S}$ with drain voltage $\left(V_{D S}\right)$. Results demonstrates higher output conductance for DMSGODP-TFET due to higher $\delta I_{D S}$ shown by DMSGO-DPTFET for the similar changes in $\delta V_{D S}$. Fig. 8(c) shows the comparative $R_{O}$ variation with $V_{D S}$ for constant $V_{G S}$. From the results, lower $R_{O}$ is observed for DMSGODP-TFET as $g_{D S}$ is higher. The variation of $C_{g s}$ and $C_{g d}$ obtained from small-signal ac analysis at $1 \mathrm{MHz}$ frequency is illustrated in Fig. 8(d) and Fig. 8(e). From the results, it has been noted that DMCG-DP-TFET shows the minimum value of $C_{g s}$, and for DMSGO-DPTFET, a significant increase of $C_{g d}$ is noted above $V_{G S}$ $=0.85 \mathrm{~V}$.

Another critical parameter for RF performance analysis of the device is cutoff frequency $\left(f_{T}\right)$. It is the frequency at which the current gain becomes unity [24]. Fig. 8(f) depicts $f_{T}$ variation with $V_{G S}$ for DMCGTFET, DMCG-DP-TFET, DMSGO-TFET and DMSGODP-TFET. Results demonstrates that, initially, $f_{T}$ increases with $V_{G S}$ due to an increase in $g_{m}$, then decreases with $V_{G S}$ after reaching the peak value due to an increase in $C_{g d}$ and decrease in $g_{m}$ due to mobility degradation. From the results, a higher $f_{T}$ is obtained for DMSGO-DP-TFET which indicates that the proposed device structure is more favourable for radio fre- quency applications. The $f_{T}$ of the device is formulated as per the expression [24].

$f_{T}=\frac{g_{m}}{2 \pi\left(C_{g s}+C_{g d}\right)}$

GBP is another important parameter for the utility of device in wide range applications, for a specified dc voltage gain of 10 , it is calculated using the expression [24].

$G B P=\frac{g_{m}}{2 \pi 10 C_{g d}}$

The GBP variations with $V_{G S}$ for DMCG-TFET, DMCGDP-TFET, DMSGO-TFET and DMSGO-DP-TFET are illustrated in Fig. 9(a). Results demonstrate that, GBP initially increases as $V_{G S}$ increases, but it decreases for higher $V_{G S}$ due to the similar reasons as discussed earlier for $f_{T}$. Also, maximum GBP is noted for DMSGODP-TFET compared to other device structures. The TGF parameter indicates how efficiently the current reaches a certain transconductance value. In this regard, Fig. 9(b) shows the comparative TGF variation with $V_{G S}$. From the results, for DMSGO-DP-TFET, higher TGF is observed at lower $V_{G S}$ and with increase in $V_{G S}$ it starts decreasing as the variation in $I_{D S}$ is small. The TGF is obtained as follows

$T G F=\left(\frac{g_{m}}{I_{D S}}\right)$

Fig. 9(c) shows the comparative variation of TFP with $V_{G S}$ at $V_{D S}=1 \mathrm{~V}$. From the results, it can be noted that DMSGO-DP-TFET shows higher TFP due to high $g_{m}$ and $f_{T}$. The TFP is obtained as follows

$T F P=\left(\frac{g_{m}}{I_{D S}}\right) f_{T}$

The transit time $(\tau)$ is an important parameter to assess the time requirement for the charge carriers to move from source to drain region [20]. Therefore, it illustrate the speed of the device. Fig. 9(d) depicts the comparative transit time $(\tau)$ variation with $V_{G S}$ at $V_{D S}$ $=1 \mathrm{~V}$. The results show that as $V_{G S}$ increases, transit time decreases due to increased $f_{T}$. Thus, better switching speed is observed in case of DMSGO-DP-TFET 


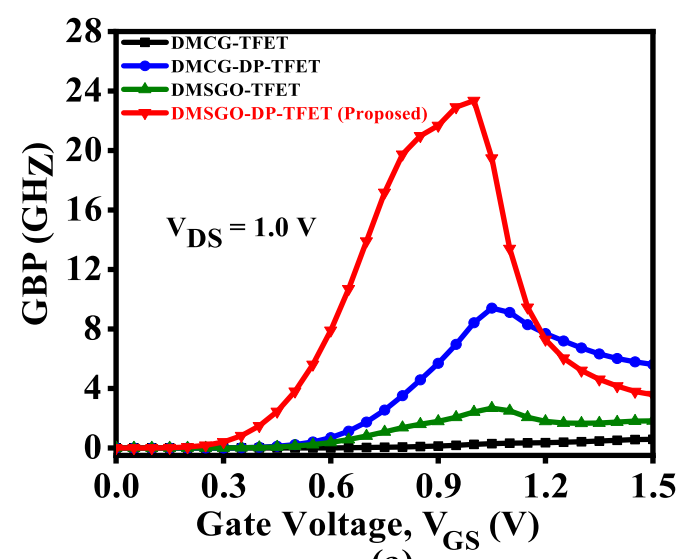

(a)

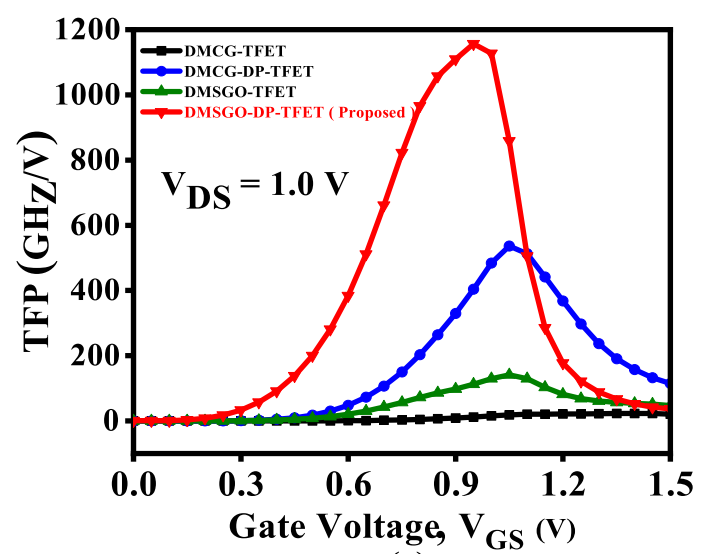

(c)

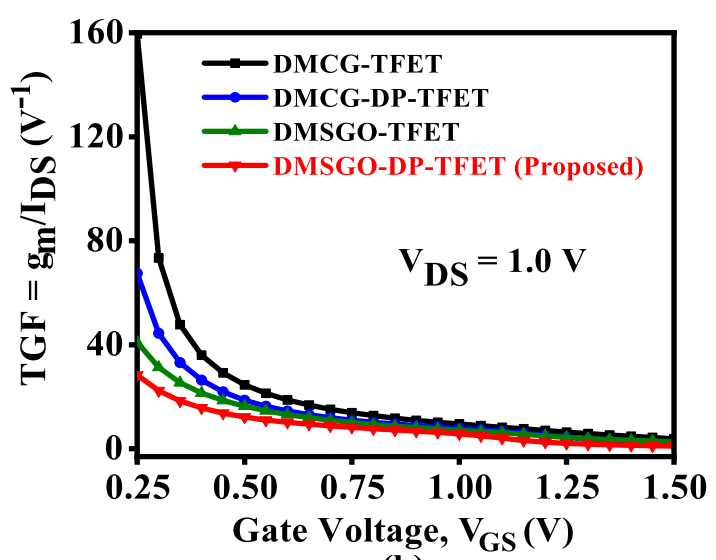

(b)

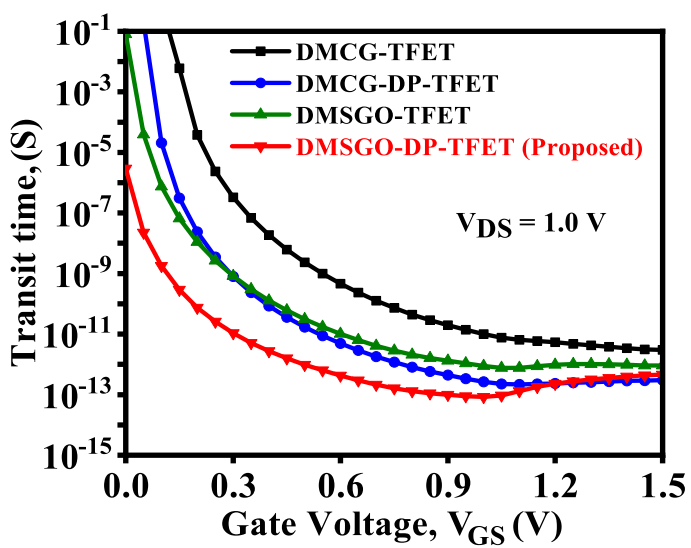

(d)

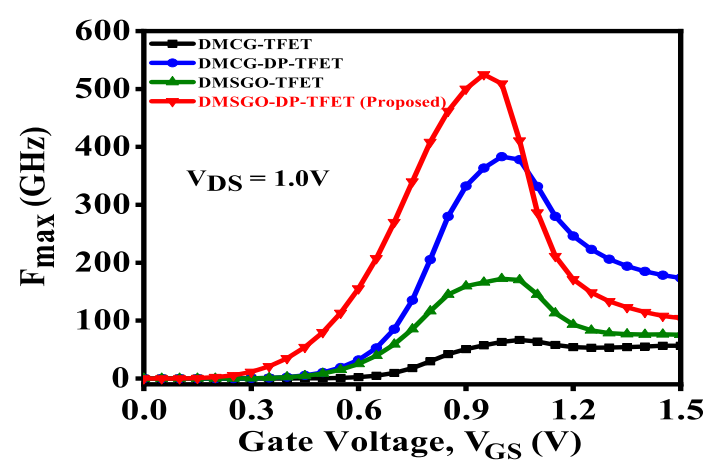

(e)

Fig. 9 Comparative performance of (a) GBP (b) TGF (c) TFP (d) Transit time (e) $F_{m a x}$ variations for DMCG -TFET, DMCG-DP-TFET, DMSGO-TFET and DMSGO-DP-TFET

as compared to DMCG-TFET, DMCG-DP-TFET, and DMSGO-TFET.

$\tau=\frac{1}{2 \pi 10 f_{T}}$

The maximum oscillating frequency $\left(f_{\max }\right)$ is another crucial parameter for radio frequency performance analysis. It is calculated by using the equation

$f_{\text {max }}=\sqrt{\frac{f_{T}}{8 \pi C_{g d} R_{g d}}}$
Here, $R_{g d}$ represents the gate resistance. Fig. 9(e) shows the $f_{\max }$ variation with $V_{G S}$ for DMCG-TFET, DMCGDP-TFET, DMSGO-TFET and DMSGO-DP-TFET at $V_{D S}=1.0 \mathrm{~V}$. From the figure, it has been noted that DMSGO-DP-TFET shows higher value of $f_{\max }$ due to higher value of $f_{T}$. 


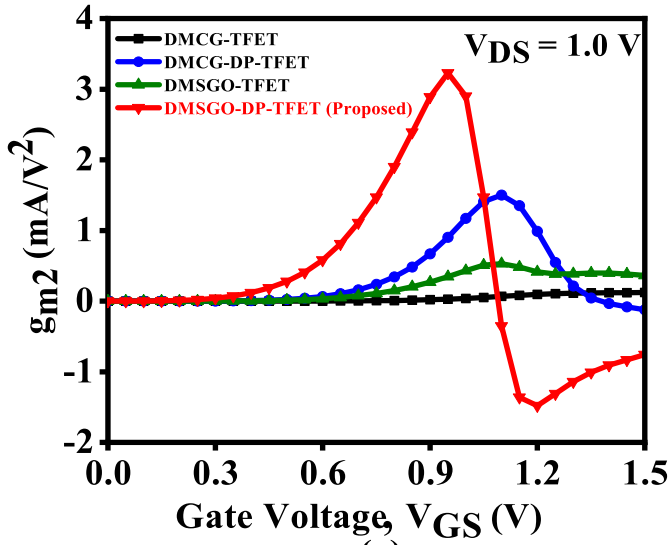

(a)

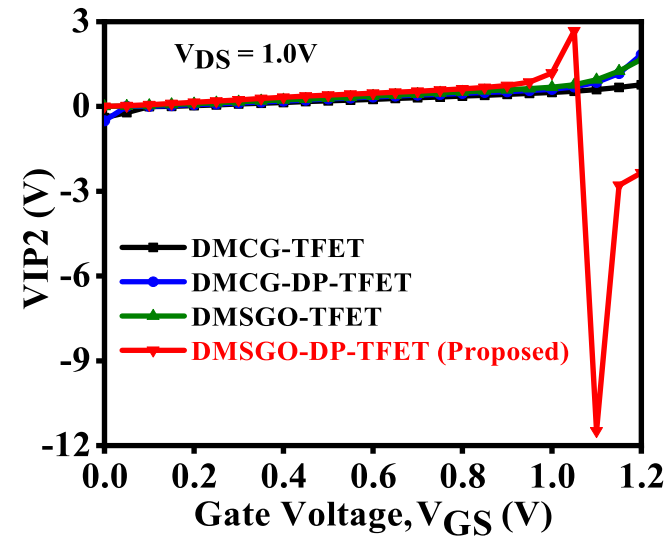

(c)

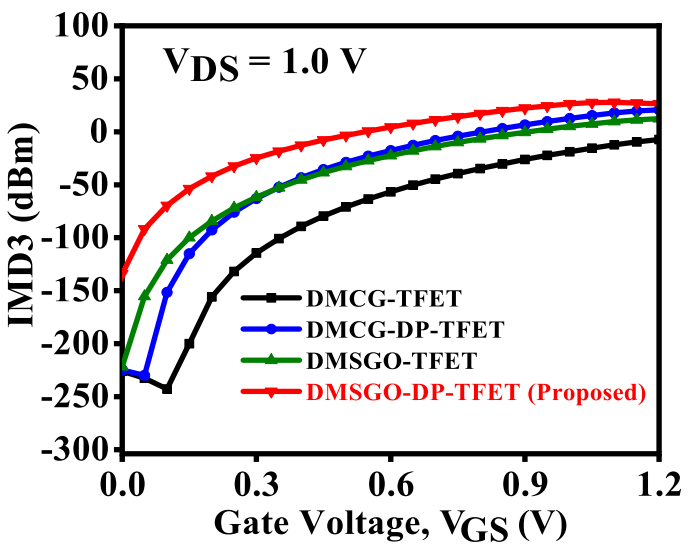

(e)

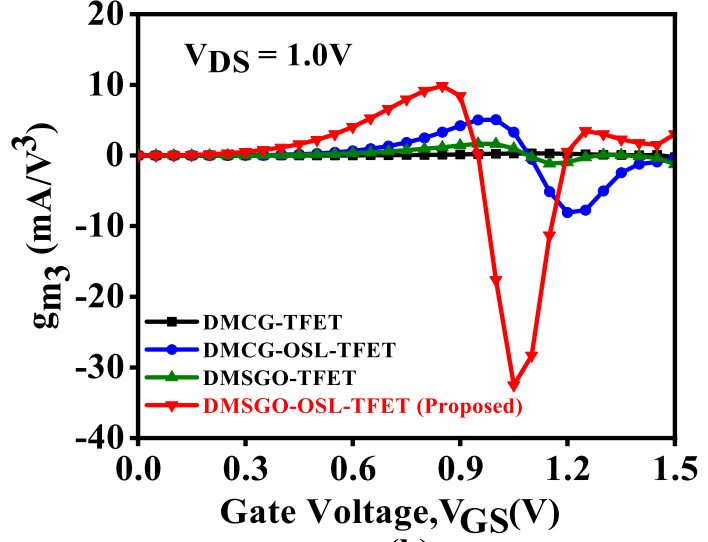

(b)

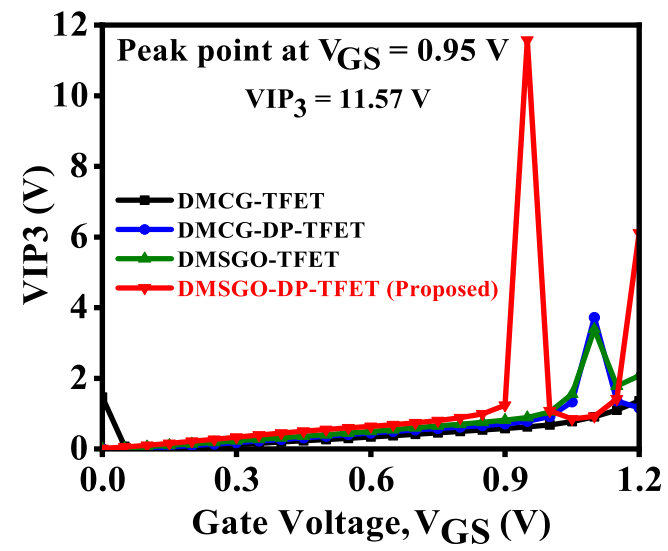

(d)

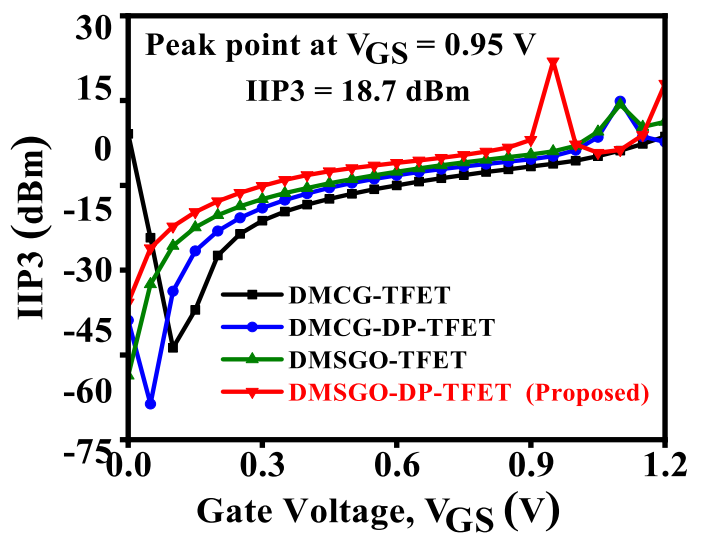

(f)

Fig. 10 Comparative performance of (a) $g_{m 2}$ (b) $g_{m 3}$ (c) VIP2 (d) VIP3 (e) IMD3 (f) IIP3 variation with $V_{G S}$ for DMCG-TFET, DMCG-DP-TFET, DMSGO-TFET and DMSGO-DP-TFET

\subsection{Linearity figure of merits}

This section presents the linearity and distortion FOMs, such as $g_{m 3}$, VIP2, VIP3, IIP3, and IMD3 for DMCGTFET, DMCG-DP-TFET, DMSGO-TFET and DMSGODP-TFET which has been explained in [25]. These are defined as follows:

$V I P 2=4 \times\left(\frac{g_{m 1}}{g_{m 2}}\right)$

$$
\begin{aligned}
& V I P 3=\sqrt{24 \times\left(\frac{g_{m 1}}{g_{m 3}}\right)} \\
& I M D 3=\left[\frac{9}{2} \times(V I P 3)^{2} \times\left(g_{m 3}\right)\right]^{2} \times R_{S} \\
& I I P 3=\frac{2}{3} \times\left(\frac{g_{m 1}}{g_{m 2} \times R_{S}}\right)
\end{aligned}
$$

Fig. 10(a) and Fig. 10(b) shows the comparative $g_{m 3}$ and $g_{m 3}$ variations with $V_{G S}$ at $V_{D S}=1.0 \mathrm{~V}$. From the 
Table 8 Performance comparison of $\mathrm{SiO}_{2}$ gate and stack gate TFET with and without dielectric pockets

\begin{tabular}{lllll}
\hline Parameters & DMCG-TFET & DMCG-DP-TFET & DMSGO-TFET & DMSGO-DP-TFET \\
\hline$I_{O N}(\mathrm{~A} / \mu m)$ & $5.02 \times 10^{-7}$ & $2.25 \times 10^{-5}$ & $1.03 \times 10^{-5}$ & $1.48 \times 10^{-4}$ \\
$I_{O F F}(\mathrm{~A} / \mu m)$ & $2.89 \times 10^{-17}$ & $3.36 \times 10^{-17}$ & $4.57 \times 10^{-17}$ & $5.22 \times 10^{-17}$ \\
$I_{O N} / I_{O F} F$ & $1.73 \times 10^{10}$ & $6.69 \times 10^{11}$ & $2.25 \times 10^{11}$ & $2.83 \times 10^{12}$ \\
$V_{T H}(\mathrm{~V})$ & 0.84 & 0.51 & 0.53 & 0.29 \\
$S S(m V /$ decade $)$ & 29.4 & 17.9 & 31.4 & 15.8 \\
Electric field $(\mathrm{MV} / \mathrm{cm})$ & 3.77 & 5.01 & 4.51 & 5.56 \\
$g_{m}(S)$ & $4.88 \times 10^{-5}$ & $5.49 \times 10^{-4}$ & $2.79 \times 10^{-4}$ & $1.01 \times 10^{-3}$ \\
$f_{T}\left(G H_{z}\right)$ & 5.34 & 81.6 & 20.8 & 193 \\
$f_{m a x}\left(G H_{z}\right)$ & 56.6 & 383 & 172 & 525 \\
GBP $\left(G H_{z} / V\right)$ & 0.579 & 10.4 & 2.69 & 23.6 \\
VIP2 $(\mathrm{V})$ & 0.77 & 1.84 & 1.66 & 2.67 \\
VIP3 $(\mathrm{V})$ & 1.35 & 3.71 & 3.38 & 11.57 \\
IIP3 $(\mathrm{dBm})$ & 0.09 & 8.849 & 8.03 & 27.75 \\
IDM3 $(\mathrm{dBm})$ & -7.19 & 20.81 & 12.42 & \\
\hline
\end{tabular}

Table 9 Performance comparison of proposed DMSGO-DP-TFET with other devices

\begin{tabular}{lllll}
\hline Possible device & $I_{O N} / I_{O F F}$ & $\mathrm{SS}(\mathrm{mV} / \mathrm{dec})$ & $g_{m}(\mathrm{mS})$ & $f_{T}(\mathrm{GHz})$ \\
\hline HGD-DW TTFET [ 12] & $9.8 \times 10^{11}$ & 18.5 & 0.290 & 59.6 \\
ED-TFET [13] & $\sim 1 \times 10^{12}$ & - & 1.02 & 190 \\
DMCG-CPTFET [14] & $\sim 6 \times 10^{12}$ & - & - & 28 \\
ICTFET [15] & $4.5 \times 10^{9}$ & - & - & 1.19 \\
MS-ED-TFET [16] & $8.92 \times 10^{8}$ & 8.07 & 0.007 & 0.17 \\
DE-QG-TFET [17] & $1.79 \times 10^{12}$ & - & 0.261 & 34 \\
DMDODG-TFET [18] & $5 \times 10^{11}$ & 18.5 & 0.09 & 8.8 \\
ESDG-TFET [19] & $2.57 \times 10^{12}$ & 12.24 & 0.238 & 37.7 \\
Proposed work & $2.83 \times 10^{12}$ & 15.8 & 1.01 & 193 \\
\hline
\end{tabular}

figure, peak values of $g_{m 3}$ and $g_{m 3}$ can be seen at lower $V_{G S}$ in DMSGO-DP-TFET as compared with other devices. A higher values of VIP2 and VIP3 is required for better linearity and minimal distortion. In this regard, Fig. 10(c) and Fig. 10(d) depicts the comparative VIP2 and VIP3 variations with $V_{G S}$ at $V_{D S}=1.0 \mathrm{~V}$. Simulation results demonstrate that VIP2 and VIP3 of DMSGO-DP-TFET is higher than the other structures. This indicates that DMSGO-DP-TFET is more linear than other devices. The next linearity and distortion performance parameter for analysis is IMD3, it originates from the non-linearity characteristics of $I_{D S^{-}}$ $V_{G S}$ and seems to be a distorting signal in wireless systems. Devices with lower IMD3 values can have the ability to withstand higher signal distortion. In this regard, Fig. 10(e) shows the comparative IMD3 variation with $V_{G S}$ at $V_{D S}=1.0 \mathrm{~V}$. From the figure, IMD3 of DMCG-TFET is noted to be smallest, indicating that the DMCG-TFET intermodulation distortion performance is the best of the above devices. The next linearity performance parameter for analysis is IIP3, for better linearity of the device IIP3 value should be high. In this regard, Fig. 10(f) shows the comparative IIP3 variation with $V_{G S}$ at $V_{D S}=1.0 \mathrm{~V}$. Results demonstrate that IIP3 for DMSGO-DP-TFET is much higher, which indicates that DMSGO-DP-TFET is more linear as compared to other structures.

\section{Conlusion}

In this work, a DMSGO-DP-TFET with dielectric pockets at the drain and source junctions is proposed. The structural parameters like gate lengths, work functions, pocket height and thickness are optimized to achieve better $I_{O N} / I_{O F F}$ ratio, reduce the ambipolar current, and improve the analog/RF performance of the device. The performance of the proposed device is analyzed using TCAD device simulator. Finally, a comparative performance analysis of the proposed device is done with other device structures and noted the combination of low-K and high-K dielectric pockets at the source and drain junctions and workfunction combination $\left(\phi_{1}=\right.$ $\left.\phi_{3}<\phi_{2}\right)$ on the stack gate oxide in the proposed device shows significant improvements in $I_{O N} / I_{O F F}, \mathrm{SS}$, $g_{m}, f_{T}, R_{O}$, transit time $(\tau)$, GBP, TGF, TFP, $f_{\text {max }}$, VIP2, VIP3, IMD3 and IIP3 given in table 8. The performance of the proposed device is compared with the recent literature shown in Table 9 which shows that the proposed device (DMSGO-DP-TFET) suitable for low power and radio frequency applications. 


\section{Declarations}

Funding: The authors did not receive any financial/funding support from any agency/organization for this work.

Conflict of Interests: No conflicts of interest. Availability of data and material (data transparency): All the data taken from another resource has been given the corresponding reference. The data, for which reference is not provided, is the original data. Code availability (software application or custom code): The code has been implemented on 2-D silvaco ATLAS device simulator.

Author contributions: Dharmender and Kaushal Nigam contributed equally on the design, analysis of the results and writing of the manuscript.

Compliance with ethical standards: The manuscript follows all the ethical standards, including plagiarism.

Consent to Participate Yes.

Consent for publication: Yes.

\section{References}

1. Kilchytska, V., Neve, A., Vancaillie, L., et al.: Influence of device engineering on the analog and RF performances of SOI MOSFETs. IEEE Transactions on Electron Devices 50(3):577-588 (2003)

2. Mohankumar, N., Syamal, B., Sarkar, C.K.: Influence of channel and gate engineering on the analog and RF performance of DG MOSFETs. IEEE Transactions on Electron Devices 57(4):820-826 (2010)

3. Young, K.K.: Short-channel effect in fully-depleted SOI MOSFETs. IEEE Transactions on Electron Devices 36(2):399-402 (1989)

4. Bangsaruntip, S., Cohen, G.M., Majumdar, A., Sleight, J.W.: Universality of short-channel effects in undopedbody silicon nanowire MOSFETs. IEEE Transactions on Electron Devices 31(9):903-905 (2010)

5. Pal, A., Sachid, A.B., Gossner, H., Rao, V.R.: Insights into the design and optimization of tunnel-FET devices and circuits.IEEE Transactions on Electron Devices 58(4):1045-1053 (2011)

6. Lee, M.J., Choi, W.Y.: Effects of device geometry on hetero-gate-dielectric tunneling field-effect transistors. IEEE Electron Device Letters 33(10):1459-1461 (2012)

7. Choi, W.Y., Park, B., Lee, J.D., Liu, T.K.: Tunneling field-effect transistors (TFETs) with subthreshold swing (SS) less than $60 \mathrm{mV} /$ dec. IEEE Electron Device Letters 28(8):743-745 (2007)

8. Boucart, K., Ionescu, A.M.: Double gate tunnel FET with high $\mathrm{k}$ gate dielectric. IEEE Transactions on Electron Devices 54(7):1725-1733 (2007)

9. Ahish, S., Sharma, D., Kumar, Y.B.N., Vasantha, M.H.: Performance Enhancement of Novel InAs/Si Hetero Double-Gate Tunnel FET Using Gaussian Doping. IEEE Transactions on Electron Devices 63(1):288-295 (2016)

10. Vijayvargiya, V., Vishvakarma, S.K.: Effect of drain doping profile on double-gate tunnel field-effect transistor and its influence on device $\mathrm{RF}$ performance. IEEE Transactions on Nanotechnology 13(5):974-981 (2014),
11. Sahay, S., Kumar, M.J.: Controlling the drain side tunneling width to reduce ambipolar current in tunnel FETs using heterodielectric BOX. IEEE Transactions on Electron Devices 62(11):3882-3886 (2015)

12. Raad, B.R., Nigam, K., Sharma, D., Kondekar, P.: Dielectric and work function engineered TFET for ambipolar suppression and RF performance enhancement. Electronics Letters 52(9):770-772 (2016)

13. Kondekar, P.N., Nigam, K., Pandey, S., Sharma, D.: Design and analysis of polarity controlled electrically doped tunnel FET with bandgap engineering for analog/RF applications. IEEE Transactions on Electron Devices 64(2):412-418 (2017)

14. Nigam, K., Pandey, S., Kondekar, P., Sharma, D., Parte, P.K.: A Barrier Controlled Charge Plasma-Based TFET With Gate Engineering for Ambipolar Suppression and $\mathrm{RF} /$ Linearity Performance Improvement. IEEE Transactions on Electron Devices 64(6):2751-2757 (2017)

15. Ashita., Loan, S.A., Rafat, M.: A high-performance inverted- C tunnel junction FET with source-channel overlap pockets. IEEE Transactions on Electron Devices 65(2):763-768 (2018)

16. Chandan, B.V., Gautami, M., Nigam, K., Sharma, D., Tikkiwal, V.A., Yadav, S., Kumar, S.: Impact of a Metalstrip on a Polarity-based Electrically Doped TFET for Improvement of DC and Analog/RF Performance. Journal of Computational Electronics 18(1):76-82 (2018)

17. Shaikh, M.R.U., Loan, S.A.: Drain-Engineered TFET With Fully Suppressed Ambipolarity for High-Frequency Application. IEEE Transactions on Electron Devices 66(4):1628-1634 (2019)

18. Kumar, S., Singh, S., Nigam, K., Tikkiwal, V.A.: Dual material dual-oxide dual gate TFET for improvement in DC characteristics, analog/RF and linearity performance. Applied Physics A 125(5):3531-3538 (2019)

19. Joshi, T., Singh, Y., Singh, B.: Extended-Source DoubleGate Tunnel FET With Improved DC and Analog/RF Performance. IEEE Transactions on Electron Devices 67(4):1873-1879 (2020)

20. Nigam, K., Kondekar, P.N., Sharma, D.: Approach for ambipolar behaviour suppression in tunnel FET by workfunction engineering. IET Micro and Nano Letters 11(8):460-464 (2016)

21. Solomon, P.M., et al.: Universal tunneling behavior in technologically relevant $\mathrm{P} / \mathrm{N}$ junction diodes. Journal of Applied Physics 95(10):5800-5812 (2004)

22. Adell, P.C., Barnaby, H.J., Schrimpf, R.D., Vermeire, B.: Band-to band tunneling (BBT) induced leakage current enhancement in irradiated fully depleted SOI devices. IEEE Trans. Nuclear Science 54(6):2174-2180 (2007)

23. Jain, P., Prabhat, V., Ghosh, B.: Dual metal-double gate tunnel field effect transistor with mono/hetero dielectric gate material. Journal of Computational Electronics, 14(2), 537-542 (2015)

24. Nigam, K., Kondekar, P.N., Sharma, D.: DC characteristics and analog/RF performance of novel polarity control GaAs-Ge based tunnel field effect of transistor. Superlattices and Microstructures 92:224-231 (2016)

25. Ghosh, P., Haldar, S., Gupta, R.S., Gupta, M.: An investigation of linearity performance and intermodulation distortion of GME CGT MOSFET for RFIC design. IEEE Transactions on Electron Devices 59(12):32633268 (2012)

26. Cui, N., Liang, R., Wang, J., Xu, J.: Lateral energy band profile modulation in tunnel field effect transistors based on gate structure engineering. AIP Advances, 2: 022111-16 (2012) 